\title{
Contemporary Issues in Depressive Disorders
}

\author{
Seon-Cheol Park ${ }^{1}$ and Yong-Ku Kim ${ }^{2}$ \\ ${ }^{1}$ Department of Psychiatry, Inje University Haeundae Paik Hospital, Busan, Republic of Korea \\ ${ }^{2}$ Department of Psychiatry, Korea University Ansan Hospital, Korea University College of Medicine, Seoul, Republic of Korea
}

Depression involves a wide range of symptoms affecting mood, cognition, and motor function domains. ${ }^{1}$ Since depression can be a cause for disability associated with mental health, treating it is regarded as a main source of medical expenditure. $^{2}$ About $30 \%$ of all depressed patients cannot achieve remission despite being fully treated with two or more antidepressants. ${ }^{3}$ Moreover, the great unmet need in the realm of psychopharmacological treatment for depression has been identified by the Sequenced Treatment Alternatives to Relieve Depression (STAR*D) study. ${ }^{4}$ This special issue aims to comprehensively review the contemporary research approaches for 1) diagnostic issues, ${ }^{5} 2$ ) pharmacogenetics, ${ }^{6}$ 3) epigenetics, ${ }^{7}$ and 4) potential neuroimaging biomarkers ${ }^{8}$ in depressive disorders.

The fourth edition (DSM-IV) to DSM-5, revision of the Diagnostic and Statistical Manual of Mental Disorders places greater emphasis on the dimensional versus the categorical approach, in defining the symptoms criteria for major depressive disorder (MDD). Thus, the dichotomization of mood disorders into bipolar affective disorder and unipolar depressive disorder, application of trans-diagnostic specifiers including "with psychotic features," "with mixed features," and "with anxious features," addition of "hopelessness" as the descriptor of a depressive mood, and elimination of "bereavement exclusion" are newly applied to define MDD in the DSM- $5 .{ }^{5}$ Furthermore, because the operational criteria for MDD consist of nine symptoms in the DSM-5, the heterogeneity of the depressive syndrome can inevitably be invoked and criticized from the viewpoint of Wittgenstein's "games" analogy., ${ }^{1,910}$ However, network analysis of symptoms in MDD patients may provide new insight regarding the definition of MDD. ${ }^{11}$ Additionally, the node and edge statistics of MDD symptoms may be supported by neurobiological underpinnings in future studies.

Gene variants coding for cytochromes that are involved in antidepressant metabolism (CYP2D6 and CYP2C19), are considered promising biomarkers based on considerable evi-

(c) This is an Open Access article distributed under the terms of the Creative Commons Attribution Non-Commercial License (https://creativecommons.org/licenses/bync/4.0) which permits unrestricted non-commercial use, distribution, and reproduction in any medium, provided the original work is properly cited. dence from pharmacogenetic studies, although clinical applications are inconclusive in terms of cost-effectiveness. ${ }^{6}$ Despite the identification of these candidate genes, no consistent genetic variants have been confirmed by the genomewide association studies (GWAS), and meta-analyses of GWAS for antidepressant response. ${ }^{12}$ Studies utilizing whole exome sequencing reported that the rs41271330 A allele in bone morphogenetic protein (BMP5), was associated a with a poorer outcome after a 12 week treatment with selective serotoinin reuptake inhibitors (SSRI), in patients with major depression. ${ }^{13}$ However, the comprehensive factors predicting an antidepressant response have not been identified by GWAS, since GWAS cannot provide a realistic model for the polygenic nature of the antidepressant response. ${ }^{6}$ The polygenic risk score (PRS), a method for estimating an individual's propensity for a specific phenotype, has been proposed as a realistic model for the polygenic nature of the antidepressant response. Nevertheless, the studies using PRS have not yet identified a reliable predictive factor for the antidepressant response, mainly due to underpowered sample size. ${ }^{14}$ Recently, machine learning has been suggested as a promising method for disentangling the complex and non-linear interactions between the gene variants involved in the antidepressant response. ${ }^{6}$

An increasing number of genes and single nucleotide polymorphisms (SNPs) associated with depression have been identified in GWAS and meta-analysis GWAS studies, and epigenetic and environmental mechanisms have been proposed as a substantial contributing factor in the pathogenesis of depression. Thus, epigenetic factors are regarded as the bridging factor between genetic and environmental mechanisms. ${ }^{15}$ The results, (which have been reproduced), demonstrated that the gene-environment $(\mathrm{GxE})$ interactions in depression are linked to loci such as CRHR1, FKBP5, HTR2A, and a serotonin transporter gene-linked polymorphic region (5-HTTLPR), in the SLC6A4 (serotonin neurotransmitter transporter) gene. ${ }^{16}$ However, in recent meta-analyses, the overall effects of GxE interactions and 5-HTTLPR in the development of depression were not confirmed. ${ }^{17-19}$ An associa- 
tion between depression and the epigenetic mechanism of DNA methylation in the BDNF, NR3C1, OXTR, and SCL6A4 genes was proposed by recent studies. ${ }^{7}$ Additionally, a longterm adaptive role of histone acetylation with respect to stress and the antidepressant response, has been suggested. ${ }^{11}$ Artificial intelligence and machine learning are also considered promising methods to validate and replicate the results from epigenetic and GxE interactions in the prediction of the antidepressant response and the diagnosis of depressive disorder. ${ }^{7}$

Neuroimaging studies (that have been replicated), demonstrated that the fronto-limbic network is associated with a promising neuroimaging biomarker of depression. ${ }^{5}$ In particular, magnetic resonance imaging (MRI) and magnetic resonance spectroscopy (MRS) data revealed that the anterior cingulate cortex (ACC), dorsolateral prefrontal cortex (DLPFC), and orbitofrontal cortex (OFC), could be the contributing regions related to the inhibition of emotional response and memory, through cognitive control. Emotional response and memory are also associated with the limbic area, including the amygdala and hippocampus. ${ }^{8}$ Thus, the structure and function of the ACC, DLPFC, OFC, amygdala, and hippocampus have been indicated as predictive factors for an antidepressant response in patients with major depression. Also, a study utilizing magnetoencephalography (MEG) reported that MDD symptoms were associated with enhanced bottom-up signals from the amygdala-ACC and ACC-DLPFC, followed by the DLPFC's inability to inhibit the excitatory signals of the amygdala. ${ }^{20}$ Despite the methodological limitations of electroencephalography (EEG), the $\theta$ density, $\theta$ cordance, a rhythm asymmetry, $\delta$ rhythm asymmetry, and characteristic sleep EEG pattern have also been proposed as biomarkers for MDD patients and for the prediction of treatment response in MDD. ${ }^{5}$ Moreover, positron emission tomography (PET) studies showed that the ACC and other prefrontal regions may be regarded as biomarkers of major depression in validation of the neuroinflammatory theory, treatment response to deep brain stimulation, antidepressants, psychotherapy, seasonal effects, and postpartum effects. $^{5}$

Although, in terms of pharmacogenetics, epigenetics, and neuroimaging (the state of the art methods generally applied), the collective research on the definition of MDD and prediction of treatment response in depressed patients is still inconclusive and inconsistent. In particular, the heterogeneity of the depressive syndrome may contribute to the irreproducible outcomes from neurobiological studies regarding the diagnosis of major depression and prediction of the antidepressant response. We speculate that network analysis results supported by neurobiological underpinnings may enable the establishment of promising biomarkers of the depressive syndrome in terms of "seeking a brain-based classification of mental illness."21

\section{Acknowledgments}

This work was supported by the National Research Foundation of Korea (NRF) grant funded by the Korea government (MSIT) (2019R1A2C1090146).

\section{ORCID iDs}

Seon-Cheol Park https://orcid.org/0000-0003-3691-4624

Yong-Ku Kim https://orcid.org/0000-0001-5694-7840

\section{REFERENCES}

1. Østergaard SD, Jensen SOW, Bech P. The heterogeneity of the depressive syndrome: when numbers get serious. Acta Psychiatr Scand 2011; 124:495-496.

2. Hockenberry JM, Joski P, Yarbrough C, Druss BG. Trends in treatment and spending for patients receiving outpatient treatment of depression in the United States, 1998-2015. JAMA Psychiatry 2019;76:810-817.

3. Sackeim HA. The definition and meaning of treatment-resistant depression. J Clin Psychiatry 2001;62(Suppl 16):10-17.

4. Deakin J, O'Loughlin C. STAR*D: a summary and UK perspective. J Psychopharmacol 2009;23:605-612.

5. Park SC, Kim YK. Diagnostic issues of depressive disorders from Kraepelinian dualism to the Diagnostic and Statistical Manual of Mental Disorders, fifth edition. Psychiatry Investig 2019;16:636-644.

6. Corponi F, Fabbri C, Serretti A. Pharmacogenetics and depression: a critical perspective. Psychiatry Investig 2019;16:645-653.

7. Tsai JS, Lin E. Epigenetics and depression: an update. Psychiatry Investig 2019;16:654-661.

8. Lai CH. Promising neuroimaging biomarker in depression. Psychiatry Investig 2019;16:662-670.

9. Zimmerman M, Galione JN, Chelminski I, McGlinchery JB, Young D, Dalrymple K, et al. A simpler definition of major depressive disorder. Psychol Med 2010;40:451-457.

10. Rosenman S, Nasti J. Psychiatric diagnoses are not mental processes: Wittgenstein on conceptual confusion. Aust N Z J Psychiatry 2012;46: 1046-1052.

11. Fried EI, Epskamp S, Nesse RM, Tuerlinckx F, Borsboom D. What are 'good' depressive symptoms? Comparing the centrality of DSM and non-DSM symptoms of depression in a network analysis. J Affect Disord 2016;189:314-320.

12. GENDEP Investigators, MARS Investigators, $S T A{ }^{\star} D$ Investigators. Common genetic variation and antidepressant efficacy in major depressive disorder: a meta-analysis of three genome-wide pharmacogenetic studies. Am J Psychiatry 2013;170:207-217.

13. Tammiste A, Jiang T, Fischer K, Magi R, Krjutskov K, Pettai K, et al. Whole-exome sequencing identifies a polymorphism in the BMP5 gene associated with SSRI treatment response in major depression. J Psychopharmacol 2013;27:915-920.

14. Shi J, Park JH, Duan J, Berndt ST, Moy W, Yu K, et al. Winner's curse correction and variable thresholding improve performance of polygenic risk modeling based on genome-wide association study summary-level data. PLoS Genet 2016;12:e1006493.

15. Howard DM, Adams MJ, Clarke TK, Hafferty JD, Gibson J, Shirali M, et al. Genome-wide meta-analysis of depression identifies 102 independent variants and highlights the importance of the prefrontal brain regions. Nature Neurosci 2019;22:343-352.

16. Sharma S, Powers A, Bradley B, Ressler KJ. Gene x environment determinants of stress and anxiety-related disorders. Ann Rev Psychol 2016; 67:239-261.

17. Culverhouse RC, Saccone NL, Horton AC, Ma Y, Anstey KJ, Banaschewski $\mathrm{T}$, et al. Collaborative meta-analysis finds no evidence of a strong interaction between stress and 5-HTTLPR genotype contribut- 
ing to the development of depression. Mol Psychiatry 2018;23:133-142.

18. Heim C, Binder EB. Current research trends in early life stress and depression: Review of human studies on sensitive periods, gene-environment interactions, and epigenetics. Exp Neurol 2012;233:102-111.

19. Covington III HE, Vialou VF, LaPlant Q, Ohnishi YN, Nestler EJ. Hippocampal-dependent antidepressant-like activity of histone deacetylase inhibition. Neurosci Lett 2011;493:122-126.
20. Lu Q, Li H, Luo G, Wang Y, Tang H, Han L, et al. Impaired prefrontalamygdala effective connectivity is responsible for the dysfunction of emotion process in major depressive disorder: a dynamic causal modeling study on MEG. Neurosci Lett 2012;523:125-130.

21. Miller G. Beyond DSM: seeking a brain-based classification of mental illness. Science 2010;327:1427. 